\section{McCune-Albright Syndrome}

Jacqueline E. van der Wal

Department of Pathology, The Netherlands Cancer Institute/Antoni van Leeuwenhoek Hospital, Amsterdam, The Netherlands

\section{Description}

Polyostotic fibrous dysplasia combined with café au lait pigmentation of the skin and oral mucosa and multiple endocrinopathies, such as sexual precocity, pituitary adenoma, or hyperthyroidism.

\section{Cross-References}

Polyostotic fibrous dysplasia
Melanosis, Smoker's 